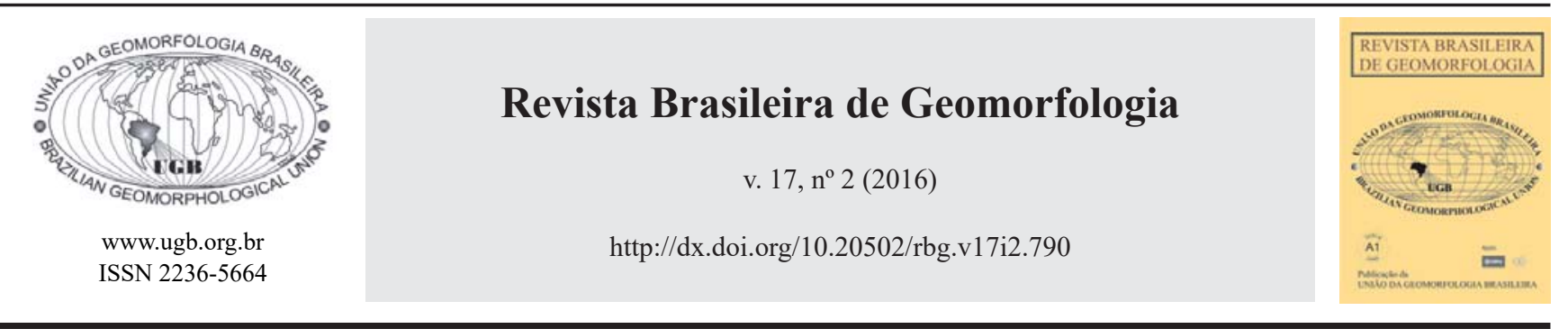

\title{
MORFOMETRIA DA BACIA DO RIO SÃO JOÃO, MG: UMA PROPOSTA DE ENTENDIMENTO DOS LIMITES E DA DINÂMICA DAS SUPERFÍCIES EROSIVAS
}

\section{MORPHOMETRY OF THE RIVER BASIN SÃO JOÃO, MG : A PROPOSAL FOR UNDERSTANDING THE LIMITS AND THE DYNAMICS OF EROSIVE SURFACES}

Samia Passarella

Instituto de Geociências, Universidade Estadual de Campinas R. João Pandiá Calógeras, 51, Campinas, São Paulo, CEP: 13083-870, Brasil

Email: passarellasm@gmail.com

\section{Francisco Sergio Bernardes Ladeira \\ Instituto de Geociências, Universidade Estadual de Campinas} R. João Pandiá Calógeras, 51, Campinas, São Paulo, CEP: 13083-870, Brasil Email: fsbladeira@ige.unicamp.br

Veraldo Liesenberg

Centro de Ciências Agroveterinárias, Universidade do Estado de Santa Catarina Av. Luís de Camões, 2090, Lages, Santa Catarina, CEP: 88520-000, Brasil

Email:veraldo@gmail.com

\begin{tabular}{l} 
Informações sobre o Artigo \\
\hline Recebido (Received): \\
29/12/2015 \\
Aceito (Accepted): \\
14/03/2016
\end{tabular}

Palavras-chave:

Parâmetros Morfométricos;

Superfícies Erosivas; Bacia do Rio São João.

\section{Keywords:}

Morphometric Parameters; Erosive Surfaces; São João River Basin.

\section{Resumo:}

Os parâmetros morfométricos da superfície do relevo são essenciais para a caracterização e o entendimento da formação de uma paisagem, especialmente em áreas que passaram por processos erosivos e tectônicos bem marcados, como o caso da região da Bacia do Rio São João, no sudoeste de Minas Gerais. Com o propósito de reconhecer os traços tectônicos que pudessem interferir no desenvolvimento do canal e por fim afetar os ciclos erosivos, foram realizadas uma série de análises geomorfológicas; tais como a Isobase, o Fator de simetria topográfica transversal com assimetria da bacia de drenagem e a Curva e integral hipsométrica. Estes índeces foram produzidos através do software TecDEM, que compilou em sua interfacie de trabalho todo o procedimento e rotina, utilizando-se apenas das informações espaciais do modelo digital de elevação (SRTM). Estas análises, favoreceram satisfatoriamente o entendimento das estruturas e do relevo no contexto da bacia, assim evidenciando os domínios topográficos e limites das superfícies, a dinâmica erosiva, o deslocamento dos canais pertencentes as bacias de quarta e quinta ordem, e por fim o momento de transição do relevo. 


\begin{abstract}
:
The morphometric parameters of relief surface are essential for characterizing and understanding the landscape formation, especially in areas that have been undergone erosion and tectonic processes, as the case of the São João River Basin in southwest of Minas Gerais state. In order to recognize tectonic features that could interpose the development of the channel and ultimately affect the erosion cycles, were performed a series of geomorphological analysis, such as: isobase, factor transverse topographic symmetry and asymmetry of the drainage basin, and the curve and hypsometric integral. These results were produced through rates obtained by the TecDEM, software used to compile the entire procedure and routine, just using spatial information of the digital elevation model (SRTM). These analyzes satisfactorily gave the understanding of the structures and relief in the basin context, and thus, showing the topography and boundary areas, the erosive dynamic displacement of channels belonging to fourth and fifth basins orders, and finally the moment of transition relief.
\end{abstract}

\section{Introdução}

Estudos de parâmetros morfométricos são essenciais para o entendimento da paisagem geomorfológica, sua evolução e dinâmica, pois fornecem o reconhecimento de elementos geomórficos típicos para a caracterização de áreas sob a influência de atividades de natureza tectônica e a possibilidade de distinguí-las das erosivas. Desde a década de 1960 com as primeiras análises e produtos feitos por Filosofov (1960), estes parâmetros têm sido constantemente aprimorados com diferentes metodologias multidisciplinares, conforme (HOWARD, 1968; MORISAWA \& HACK, 1985; SUMMERFIELD 1987, 1991; SCHUMM, 1981, 1986, 2000, 2007; COX, 1994; BURBANK \& ANDERSON, 2001).

Os sensores remotos, ao gerarem informações mais completas da superfície terrestre, em especial a geração de modelos digitais de elevação (MDE), proporcionaram o desenvolvimento de análises cada vez mais refinadas, com informações espaciais de latitude, longitude e altitude. Estes dados tornaram possível a implementação de ferramentas capazes de cobrir, dos mais antigos aos mais recentes fenômenos tectônicos nos mais variados ambientes. Exemplos de aplicações geomorfológicas e tectônica usando dados remotamente situados podem ser encontrados em (GARROTE et al., 2008; GROHMANN et al., 2007, 2011; SHAHZAD et al., 2009; SHAHZAD \& GLOAGUEN, $2011 \mathrm{a}, \mathrm{b}$; KIRBY \& WHIPPLE, 2001).

Neste trabalho, foram usadas as análises morfométricas de Isobase, Fator de simetria topográfica transversal e Assimetria da bacia de drenagem e Integral hipsométrica, com o objetivo de reconhecimento do controle de estruturas sobre do relevo da Bacia do Rio São João, Figura 1. Também foi objetivo identificar a sua relação com as superfícies erosivas dispostas na área que foi afetada por processos intensos de denudação associados a soerguimento de arcos, deposição de sedimentos em bacias marginais e formação de bacias continentais, com diferentes ciclos erosivos.

\section{2. Área de estudo}

A região de estudo está localizada na Plataforma Sul-Americana, porção sudeste do Brasil, sendo composta por núcleos pré-cambrianos relacionados com áreas cratônicas e faixas de dobramentos Almeida et al., (1977), Figura 2.

A unidade geológica mais antiga compreende o Cráton do São Francisco, caracterizado por embasamento arqueano exposto, composto por gnaisse, migmatito e anfibolito, cujos protólitos se caracterizam por idades entre 3,2 e 2,9 Ga, segundo, (CAMPOS NETO, 2000 e BARBOSA \& SABATÉ, 2004).

A Faixa Brasília compreende uma zona de faixas de dobramentos, onde seu setor meridional é marcado por empilhamento de terrenos e escamas de empurrão (Figura 2). As principais unidades compreendem rochas metassedimentares que foram afetadas por diversos processos de metamorfismo. Destacam-se na área estudada o Grupo Canastra, Ibiá, Grupo Araxá, e Andrelândia (VALERIANO et al., 2004).

Episódios magmáticos cretáceos ocorreram na região no início da abertura do Atlântico a partir da quebra do supercontinente Gondwana há aproximadamente 130 Ma e.g., (ALKMIM \& MARTINS-NETO, 2001; CONDIE, 2005; HASUI, 2010, TELLO et al., 2003; TELLO et al., (2005); SOARES et al., 2013). Neste processo destaca-se: (1) o magmatismo basáltico na Bacia do Paraná entre 130-120 Ma (MARQUEs \& 


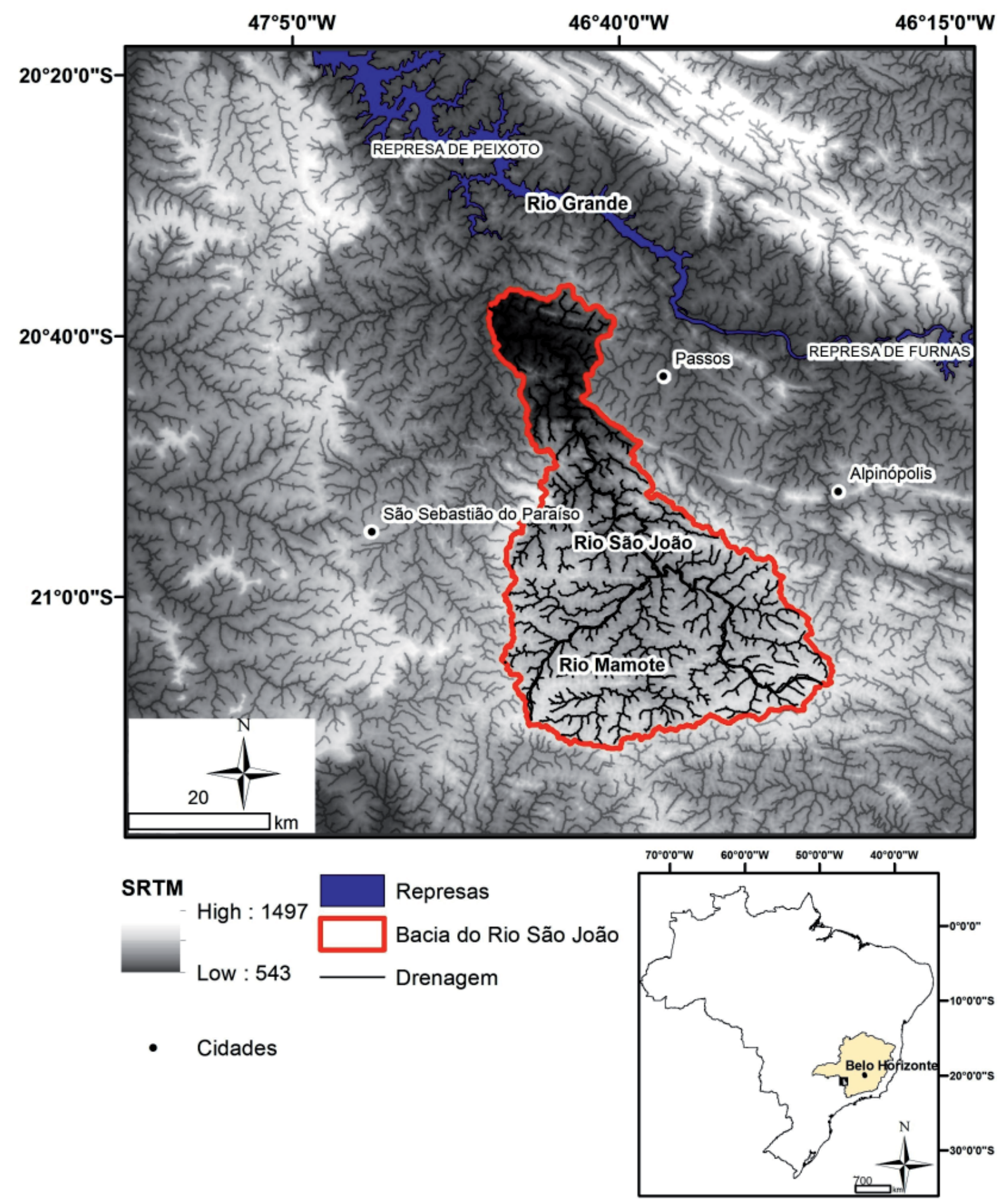

Figura 1 - Localização e contexto da área estudada.

ERNESTO, 2004); (2) os complexos alcalino-carbonatíticos de Araxá, Tapira e Poços de Caldas que intrudem rochas metamórficas neoproterozóicas da Faixa Brasília, Soares et al., (2013); e (3) rochas ultrabásicas potássicas e ultrapotássicas com afinidades kamafugíticas, representadas pelo Grupo Mata da Corda (LEONARDOS \& ULBRICH, 1991; ALKMIM, 2004).
De acordo com Hasui (2010), esta região passou por um perído de estabilidade tectônica entre o final do Cretáceo e o início do Paleógeno, originando a Superfície Sul-Americana. Atualmente, esta superfície está associada a topos aplainados a altitudes entre 1100 e $1200 \mathrm{~m}$.

A estabilidade tectônica posterior à ruptura do 
supercontinente Pangéia favoreceu a formação de coberturas detrito-lateríticas ferruginosas do Paleógeno ao Quaternário que se encontram sobrepostas aos demais grupos rochosos, Hasui (2010) (Figura 2).

\section{Metodologia}

Foram aplicadas as técnicas morfométricas de Isobase, Fator de simetria topográfica transversal e da bacia e integral hipsométrica, através do ferramental do software MATLAB o TecDEM, (SHAHZAD \& GLOAGUEN, $2011 \mathrm{a}, \mathrm{b}$ ), com MDE (Modelo Digital de Elevação), com resolução espacial de 90 m, trabalhados a partir da base de dados SRTM (Shuttle Radar Topographic Mission, FARR et al., 2007). Todos os produtos gerados para o estudo da morfotectônica foram extraídos apartir deste do MDE, tabela 1.

\section{a-Isobase}

A isobase é considerada uma superfície hipotética determinada pela intersecção entre drenagens de ordem similar, com a topografia associada (FILOSOFOV, 1960), é uma representação simplificada mediante o

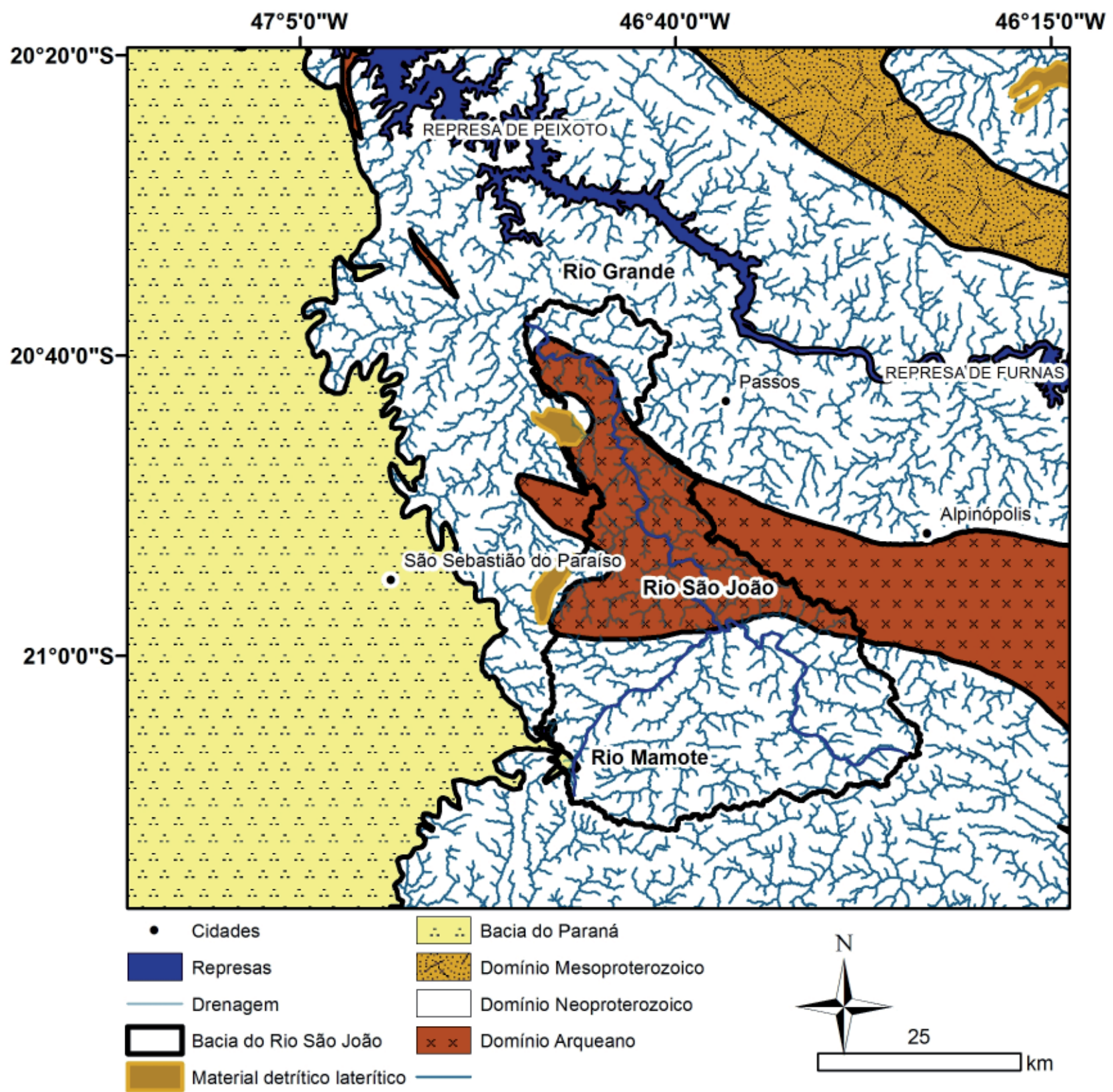

Figura 2 - Mapa geológico regional simplificado, extraído da base de dados da CPRM. 
Tabela1: Parâmetros morfométricos

\begin{tabular}{|c|c|c|}
\hline Técnica & Propósito & $\begin{array}{l}\text { Unidade } \\
\text { trabalhada }\end{array}$ \\
\hline a-Isobase e knicpoints & $\begin{array}{l}\text { Compor uma melhor delimitação das diferentes superfices } \\
\text { erosivas. }\end{array}$ & km \\
\hline $\begin{array}{c}\text { bFator de simetria } \\
\text { topográfica transversal e } \\
\text { Assimetria da bacia de } \\
\text { drenagem }\end{array}$ & $\begin{array}{l}\text { Verificar o comportamento do deslocamento do canal e da bacia a } \\
\text { cada } 2 \mathrm{~km} \text {. }\end{array}$ & $K m$ \\
\hline c-Integral Hipsométrica & $\begin{array}{l}\text { Caracterizar o nível de desenvolvimento do relevo mediante a } \\
\text { relação da altura pela área do canal }\end{array}$ & $K m$ \\
\hline
\end{tabular}

relevo original, Steiner, (2007), que assim permite classificar num DEM, zonas que compõe em um relevo homogêneo a partir de uma determinada ordem de drenagem. O produto gerado permite uma comparação entre as superfícies mais elevadas que representam as formas de relevo antes da dissecação dos canais, da ordem selecionada para comparação, e as zonas mais dissecadas, que sofreram com a atuação dos mesmos. No caso deste trabalho os canais selecionados se encontram acima da segunda ordem.

\section{b- Fator de simetria topográfica transversal e Assimetria da bacia de drenagem}

O fator de simetria topográfica transversal (FSTT), quantifica numa escala de 0 a 1 a direção da migração média dos canais, considerando que a migração preferencial do canal indica a assimetria do perfil topográfico transversal ao seu eixo, através da relação:

$$
T=D a / D d
$$

onde $\mathbf{T}$ corresponderia ao fator de simetria, Da, seria a distância da linha média do eixo da bacia de drenagem até a linha média do cinturão do meandro ativo e Dd a distância da linha média da bacia ao divisor da bacia.

Essa relação permite uma discriminação entre migração de canais como uma consequência de processos internos fluviais (valores próximos de 0 ) ou de forças tectônicas (valores próximos de 1), Cox (1994), Cox et al., (2001), Garrote et al. (2008).

Já o fator de assimetria da bacia de drenagem (FABD), Hare \& Gardner, (1985), Keller \& Pinter (1996), Salamuni (1998), apresenta o componente de migração perpedicular ao seu eixo que pode ser resultado de processos fluviais internos, com valores iguais ou próximos a 50, ou processos fluviais sucetíveis a forças externas que no caso, menores que 50 indicam um basculamento do canal na sua margem esquerda, se maior que 50 demonstram provável basculamento na margem direita.

Esses valores são obtidos através da equação:

$$
\text { FA }=100(\mathbf{A r} / \mathbf{A t})
$$

onde FA seria o fator de assimetria, Ar, corresponderia $\mathrm{a}$ área da bacia à direita do rio (olhando ajusante), At a área total da bacia de drenagem.

\section{c-Curva e Integral Hipsométrica}

A curva hipsométrica é a representação gráfica do relevo de uma bacia hidrográfica. Essa representação se dá através da integral Hipsométrica $(\mathrm{Hi})$, índice que descreve a "curva" de distribuição de elevação do terreno de uma determinada área da paisagem por meio da frequência acumulada das altitudes (CHORLEY \& MORLEY, 1959; STRAHLER, 1957; SCHUMM, 1981, 1986, 2000 e 2007). Esse índice (Hi) em termos 
quantitativos, representa porções do relevo que ainda não foram sujeitas ao rebaixamento erosivo e por isso pode indicar diversos estágios evolutivos da paisagem (KELLER E PINTER, 1996; PÉREZ-PEÑA et al., 2009; ZANI et al.2010).

Portanto cada curva obtida por essa técnica representa a proporção total da altura da bacia $(\mathbf{h} / \mathbf{H})$ pela proporção total de sua área (a/A). Cabe ressaltar também que todas as equações utilizadas para determinar integral da curva hipsométrica no software TecDEM, (SHAHZAD \& GLOAGUEN, $2011 \mathrm{a}, \mathrm{b}$ ) foram extraídas de Harlin (1978) e Pérez-Peña et al., (2009p.1216).

\section{Resultados e discussão}

\section{a-Isobase}

O mapa de isobase, representado na Figura 3, apresenta três diferentes domínios evolutivos na zona da bacia, a) domínio das antigas superfícies, b) zona de transição, c)domínio das superfíces recentes.

O primeiro domínio, (a)domínio das antigas superfícies, remete às áreas mais elevadas da região, indicado pelos tons de amarelo claro a vermelho, que estão acima de 900 metros, e apresenta zonas preservadas pelo arcabousso litológico do Neoproterozóico, indicando que sua formação sucede os intensos processos metamórficos da Faixa Brasília, Figura 2. Na área, esse fato é comprovado através dos depósitos lateríticos que estão instalados a $1200 \mathrm{~m}$, recobrindo a sinforma do Morro do Chapadão, Passarella et al., (2010), Figura 4. Outro acontecimento importante é a correlação do material presente no divisor da bacia que forma um transecto com o knicpoint destacado pelo número 3, Figuras 3 e 4,assim sugerindo que aconteceu em algum momento de transição destes ciclos erosivos uma alteração do nível de base. Alguns autores dizem que neste caso o material laterítico em destaque seria um remanescente da Superfície Sul Americana, King (1956) e Valadão (1998).

O domínio (b) zona de transição, possui uma coloração verde que representa a zona de intermediária que marca a passagem do domínio de antigas superfícies preservadas para a porção mais rebaixada da topografia, nele se concentram dois knickpoints da bacia do Rio São João(1 e 2), reforçando ainda mais seu caráter de transição. Em toda sua extensão foi denudado até o embasamento cristalíno, sem preservar se quer um depósito detrítico laterítico, conferido no mapa geológico pelo domínio arqueano Figuras 2 e 3.

O domínio(c), das superfícies recentes, se encontra em tons de azul com cotas altimétricas que variam de 700 a $600 \mathrm{~m}$. Nesse patamar é possível de observar a reorganização da paisagem na construção de superfícies recentes mais próximas dos níveis de base regionais atuais. Também é possivel de reconhecer que neste trecho existiu uma forte influência tectônica que alterou toda a estrutura da bacia, mudando o rumo do curso principal assim como o basculamento do canal, que passa a alterar de forma mais agressiva, Figura 3 e Tabela 2.

\section{b- Fator de simetria topográfica transversal e Assimetria da bacia de drenagem}

A análise de simetria topográfica transversal se estabeleceu na composição de quinta ordem da bacia do Rio São João, apresentada na Tabela 2 e Figura 4.

Os canais com maiores valores de deslocamento, acíma de $0.65 \mathrm{~km}$, seguem sequencialmente do número 21 ao 29 e se apresentam no trecho que remete ao domínio (a) (domínio das antigas superfícies), nele as estruturas marcadas por falhamentos antigos, apresenta o deslocamento obedecendo ao desenho das escamas de empurrão da Faixa Brasília, de escala crustal que convergiram para leste, contra o Cráton do São Francisco, durante o Ciclo Brasiliano (Neoproterozóico) entre 780 a 640 Ma, Morales et al. (1993), Heilbron et al., (1987); Simões,(1993).

O nível de deslocamento intermediário é representado pelos canais 12 ao 18 que são aqueles que estão entre os limítes dos domínios geológicos, Neoproterozóico e Arqueano, o que pode indicar que ainda existe influência nestes canais das atividades tectônicas destacadas no parágrafo acima, Tabela 2.

No entanto para os canais de $11,12,13$ essa influencia não seria dominante, pois já se encotram bem estabelecidos no domínio Arqueano.

Fica nítido tanto pela Figura 4 tanto quanto pela Tabela 2 que o basculamento preferencial dos canais segue para a direita, onde todos eles se apresentam com valores acima de $50 \mathrm{~km}$.

No entanto, não devemos desprezar a forte tração do basculamento para a direita, na porção mais baixa da bacia, que representa de forma clara a atividade tectônica que recotou o trecho em duas porções totalmente diferentes em termos de direção. 


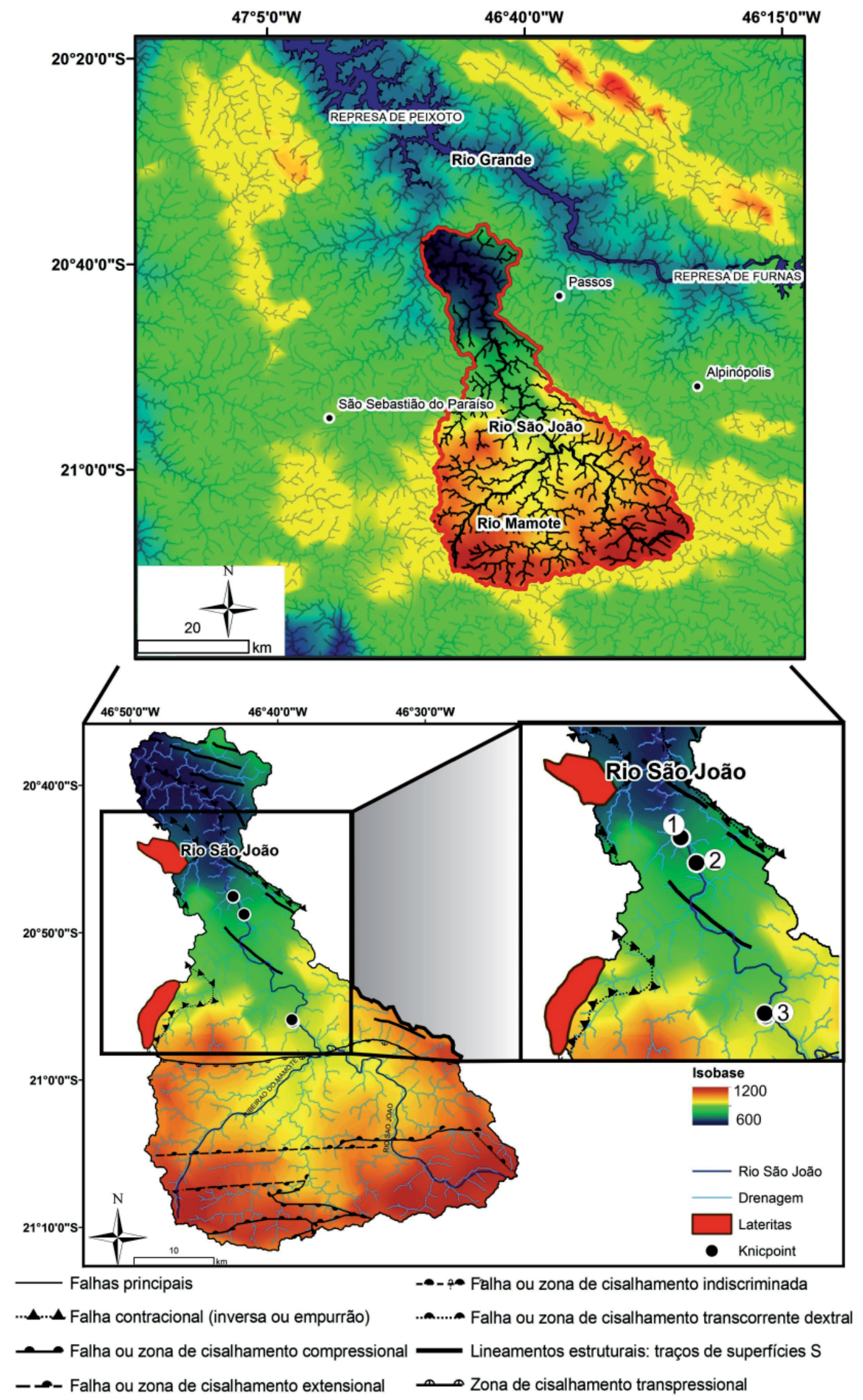

Figura 3 - Mapas de isobase e knicpoints, com os principais lineamentos e materiais detríticos lateríticos, (extraídos da base de dados CPRM, Brasil ao milhonésimo) gerado a partir do modelo digital de elevação SRTM (Shuttle Radar Topographic Mission, FARR et al., 2007). 

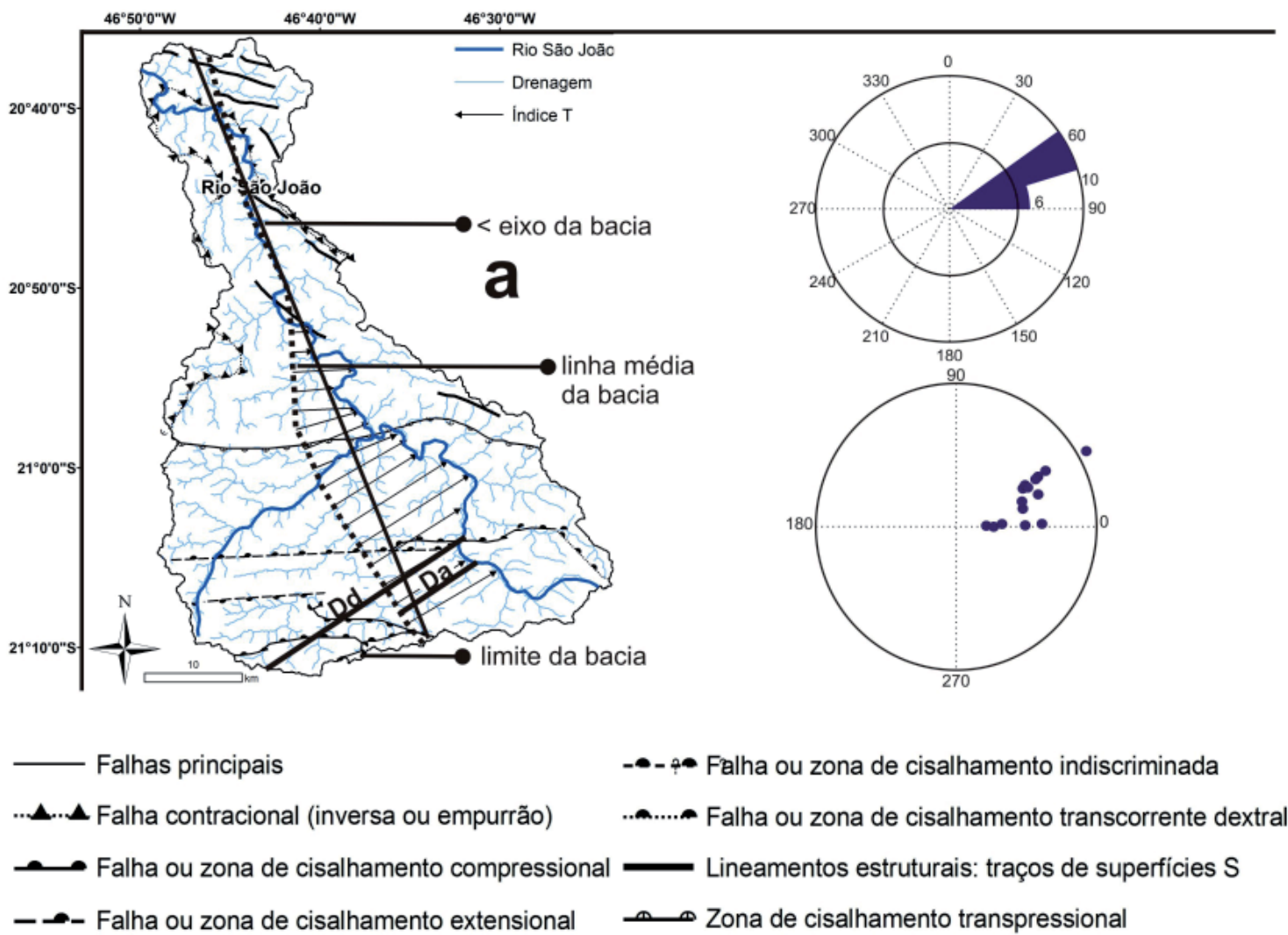

Figura 4 - Mapa do Fator de simetria topográfica transversal com os principais falhas regionais (base de dados CPRM, Brasil ao milhonésimo), junto com os diagramas de roseta e polar extraídos do canal principal do rio São João.

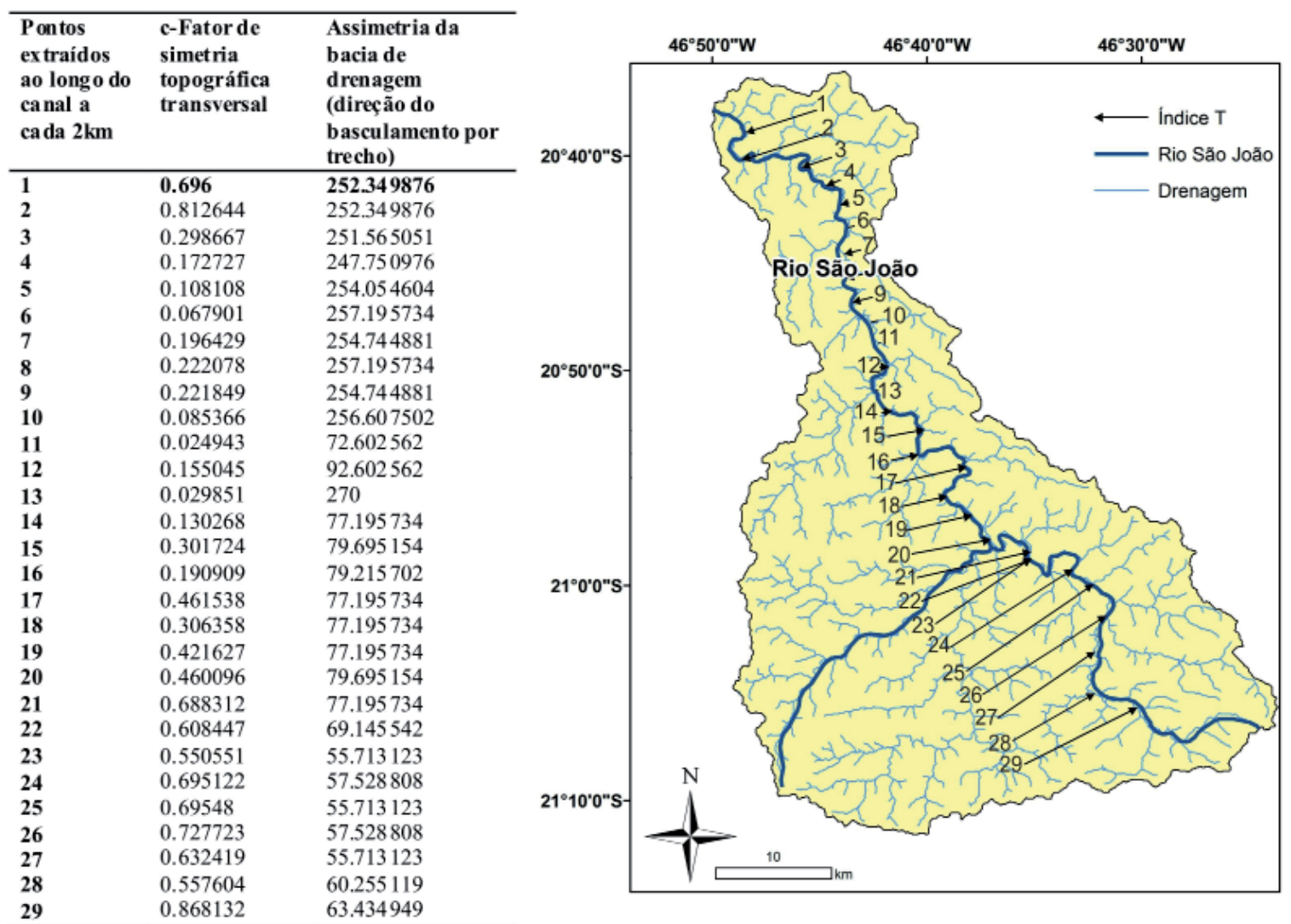

Figura 5 - Dados morfométricos do Fator de simetria topográfica transversal e Assimetria da bacia de drenagem. 


\section{c-Curva e Integral Hipsométrica}

A curva de integral hipsométrica, apresenta um reforço aos dados da isobase, pois representa de forma gráfica a disposição dos três domínios da bacia por meio da proporção total da altura X área da bacia Figura 6.

Podemos perceber num primeiro instante que a bacia majoritariamente se encontra no domínio (a), domínio das antigas superfícies,onde os canais possuem o maior índice de deslocamento em relação ao seu eixo principal para o sentido E, Figuras 4, 5 e 6 .

No entanto existe uma quebra levemente abrupta na curva que marca o momento de mudança altimétrica do canal onde ele parte para uma cota mais baixa e busca por estabilidade, que por fim, pode ser relacionada ao domínio (b) zona de transição. Até este momento nota-se que pela estabilidade da curva, a bacia ainda manteve um baixo potencial denudacional, típico de um relevo bem estabelecido na paisagem, Figuras 4, 5 e 6.

A partir dessa quebra apresenta-se um momento denudacional mais intenso, que leva o sistema a cotas altimétricas mais baixas e por isso indica um estágio de desenvolvimento de uma topografia jovem em busca de equilíbrio, possível de se relacionar com o domínio (c), domínio das superfíces recentes, Figuras 4, 5 e 6.
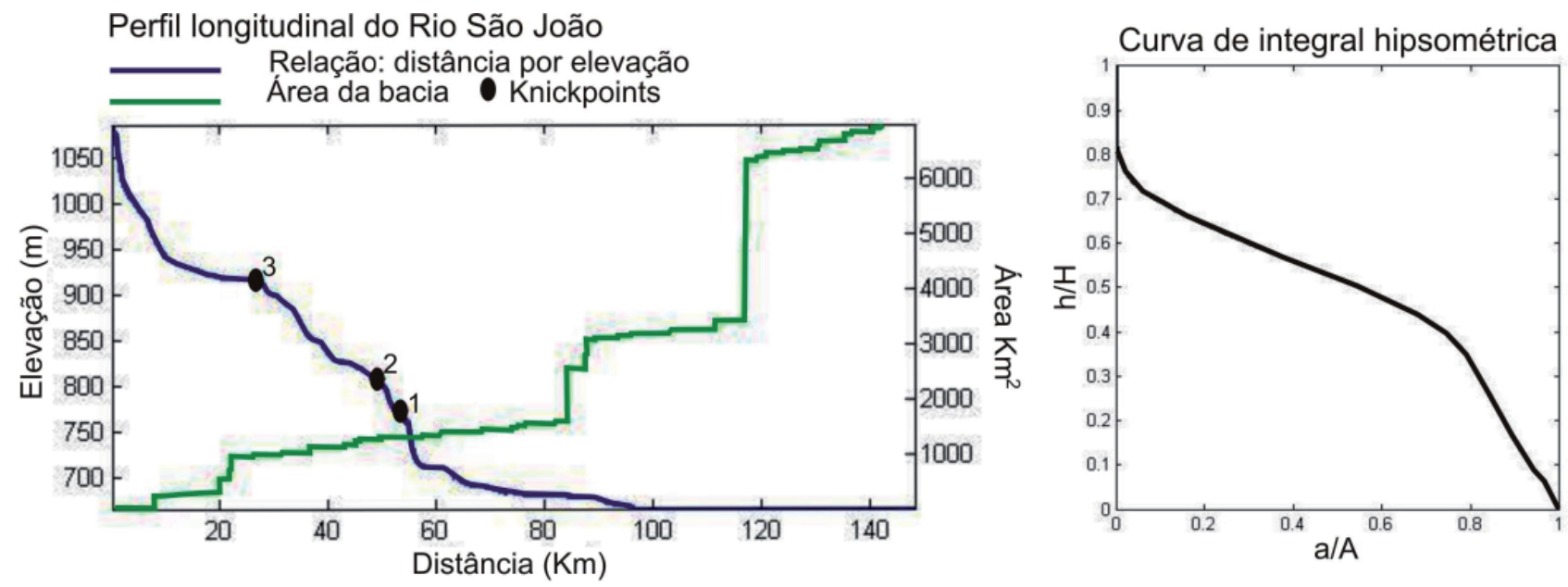

Figura 6 - Perfil longitudinal do canal e curva da Integral hipsométrica.

\section{Conclusões}

A Bacia do Rio São João possui características marcantes a respeito de seus canais, que foram fortemente condicionados pela tectônica, e posteriormente retrabalhados pelas condições climáticas. As análises de isobase, fator de simetria topográfica transversal e assimetria da bacia de drenagem e integral hipsométrica, favoreceram uma melhor compreenção a respeito da delimitação, desenvolvimento e comportamento do relevo da área investigada.

Os resultados obtidos se mostraram plausíveis com o histórico geológico e geomorfológico pré-existente, e ofereceram arcabouço ao entendimento mais refinado da dinâmica da área estudada, tanto no aspecto erosivo quanto tectônico.

O mapa de isobase, representado pela Figura 3, demarcou os 3 domínios topográficos principais.O primeiro ciclo, demarcado pelo domínio (a), antígas superfícies, remonta um momento mais antigo, que se casa com as propostas de King (1956) e Valadão (1998) que seria relativo a Superfície Sul Americana. Fato este que pode ser confirmado por uma série de premissas indicadas pelos mesmos autores, como a altimetria acima de $950 \mathrm{~m}$, onde os depósitos de materiais detríticos lateríticos, geralmente se localizam sobre as rochas Neoproterozóicas em que registram deformações como sinformas, antiformas e escarpas de falhas, o arranjo da drenagem da bacia fortemente condicionado pela estrutura litológica e o aspecto de material ser apresentado por crostas endurecidas duras e homogênias, Figuras, 2, 3 e 4.

Um segundo ciclo, demarcado pelo domínio (b) zona de transição, marca a mudança do relevo, que após a quebras dos knickpoints (1 e 2), passa rebaixar abruptamente até a fase atual. Neste domínio nota se 
que a denudação começa a se intensificar, levando e retrabalhando quase todo o material superficial que recobre o domínio Arqueano, Figuras 3 e 4.

O domínio (c), das superfícies recentes, demarca um novo cíclo erosivo que ainda passa por um processo de ajuste com a topografia atual, e boa parte dos remanescentes dele se encontram retrabalhados com mais materiais ricos em quartzo próximos as calhas fluviais do Rio Grande.

A integral e curva, hipsométrica, reforça de forma gráfica essa passagem de diferentes níveis erosivos, mostrando em sua curva que a bacia segue de um relevo aplainado e bem estabelecido para uma topografia mais acidentada. O que nos leva a "deduzir" em conjunto com os outros resultados que existe a atuação de um processo de denudação que permitiu um rebaixamento do nível de base e que pode ter ocorrido os transporte dos materiais lateríticos retrabalhados em cotas menos elevadas.

O deslocamento do canal do Rio São João, foi evidenciado pelo fator de simetria topográfica transversal e assimetria da bacia de drenagem. Esses fatores sugeriram que o deslocamento intenso do canal se deu por falhas que formaram uma zona de cizalhamento que divide a bacia da parte alta para a parte baixa, Figuras, 3,4 e 5 .

Na porção mais rebaixada nota-se um deslocamento para o sentido esquerdo mas mesmo assim com intenso basculamento para a direita (ainda mais forte que na porção sul), a medida que o canal segue para o limíte sul da bacía, correspondente ao domínio Neoproterozóico, mais intensa é a influência da atividade tectônica em seu curso com o trend preferencial de N60E. $\mathrm{O}$ deslocamento do canal neste sentido permite deduzir que a dinâmica tectônica facilitou o processo erosivo da bacia no sentido da margem direita do Rio São João, provavelmnte por possuir uma litologia mais sensível que a da margem esquerda que portanto preservou os antigos materiais lateríticos naquela porção.

\section{Agradecimentos}

A gradecemos a Fundação de Amparo à Pesquisa do Estado de São Paulo - FAPESP, processos 2010/17423-3 e 2011/233257, pelo projeto e bolsa concedida.

O segundo autor agradece a Bolsa Produtividade de Pesquisa CNPq, processo308629/2015-9.

\section{Referências Bibliográficas}

ALMEIDA, F.F.M.; HASUI, Y; NEVES, B.B.B; FUCK, R.A., 8., 1977, Campina. SIMPÓSIO DE GEOLOGIA DO NORDESTE: Atas. Campina Grande: Sbg, 1977. P. 363-391.

ALKMIM F.F. \& MARTINS-NETO M.A. A Bacia Intracratônica do São Francisco: Arcabouço Estrutural e Cenários Evolutivos. In: PINTO, C.P.; MARTINS-NETO, M.A. (Ed.). Bacia do São Francisco: Geologia e Recursos Naturais. Belo Horizonte: Sbg/mg, 2001. p. 9-30.

ALKMIM F.F. O que faz de um cráton um cráton ? O Cráton do São Francisco e as revelações Almeidianas ao delimita-lo. In: MARTINS-NETO, M.A. et al (Ed.). Geologia do Continente Sul Americano: Evolução da obra de Fernando Flávio Marques de Almeida. São Paulo: Becca, 2004. p. 17-35.

BArbosa, J. S. F. \& SABATÉ, P. Archean And Paleoproterozoic Crust Of The São Francisco Cráton, Bahia, Brazil. Geodynamic Features: Prec. Res, 2004.

B URBANK, D; ANDERSON, R. Tectonic geomorphology. Oxford: Blackwell Publishing, 2001.

CAMPOS NETO, M. C. Orogenic Systems from Southwestern Gondwana, an approach to Brasiliano-Pan African Cycle and Orogenic Collage in Southeastern Brazil. In: CORDANI, U.G.et al. Tectonic Evolution in South America. Rio de Janeiro: Becca, 2000. p. 335-365.

CHORLEY, R. J \& MORLEY, L.S.D. A Simplified Approximation for the Hypsometric Integral. Journal of Geology. Chicago, p. 566-571.1959.

CONDIE, K. C. High field strength element ratios in Archean basalts - a window to evolving sources of mantle plumes? Lithos,Amsterdam, v. 79, p.491-504, abr. 2005.

COX, RANDEL TOM. Analysis of drainage-basin symmetry as a rapid technique to identify areas of possible Quaternary tilt-block tectonics: an example from the Mississippi Embayment. Bulletin Of The Geological Society Of America, Boulder, v. 106, n. 5, p.571-581, 1994.

FARR, TOM G. et al. The Shuttle Radar Topography Mission. Rev. Geophys., [s.1.], v. 45, n. 2, 19 maio 2007. WileyBlackwell. http://dx.doi.org/10.1029/2005rg000183.

FILOSOFOV,V.P. Brief Guide to Morphometric Methods in Search of Tectonic Features. Saratov: Saratov University Publishing House, 1960.

GARROTE, J; HEYDT, G.G; COX, R.T. Multi-stream order analyses in basin asymmetry: a tool to discriminate the influence 
of neotectonicas in fluvial landscape development (Madrid Basin, Central Spain). Geomorphology, Amsterdam, v. 1, n. 102, p.130-144, 2008.

GROHMANN, C. H.; RICCOMINI, C.; CHAMANI, M. A. C.. Regional scale analysis of landform configuration with baselevel (isobase) maps. Hydrol. Earth Syst. Sci., [s.1.], v. 15, n. 5, p.1493-1504, 18 maio 2011. Copernicus GmbH. http://dx.doi. org/10.5194/hess-15-1493-2011

HARLIN, JOHN M. Statistical moments of the hypsometric curve and its density function. Statistical Moments Of The Hypsometric Curve And Its Density Function, [s.1.], v. 10, n. 1, p.59-72, jan. 1978.

W, HARE P; W, GARDNER, I. Geomorphic indicators of vertical neotectonism along converging plate margins. Nicoya Peninsula, Costa Rica. In: M, Morisawa; T, Hack J (Ed.). Tectonic Geomorphology: 15th. Annual Binghamton Geomorphology Simp. Binghamton: Procedings, 1984. p. 390.

HEILBRON, M. et al. O contato basal do Grupo Canastra entre Itaú de Minas e Carmo do Rio Claro, MG. In: SIMP. GEOL. MINAS GERAIS, 4., 1987, Belo Horizonte. Anais... Belo Horizonte: Sbg/mg, 1987. v. 6, p. 176 - 198.

HOWARD, A. D. Stratigraphic and structural controls on landform development in the Central Kentucky Karst. National Speleological Bulletin, [s.1.], v. 30, n. 1, p.95-114, jan. 1968.

KELLER, E.A; PINTER, N. Active tectonics. Santa Barbara: Prentice-hall, 1996.

KING, CHARLES LESTER. A Geomorfologia do Brasil Oriental. Revista Brasileira de Geografia, Rio de Janeiro, v. 18, n. 2, p.147-266, 1956.

LEONARDOS, H. O; ULBRICH, M. N; GASPAR, J.C. The Mata da Corda volcanic rocks. In: FIFTH INTERNATIONAL KIMBERLITE CONFERENCE, 50., 1991, Araxá. Conference. Cprm, 1991.p. 655 - 673.

MARQUES, S L; ERNESTO, M. O Magmatismo Toleítico Da Bacia Do Paraná. In: Martins-Neto, M. A. et al (Ed.). Geologia Do Continente Sul - Americano: Evolução Da Obra De Fernando Flávio Marques De Almeida. São Paulo: Becca, 2004. p. 245-263.

MORALES, NORBERTO. Evolução tectônica do cinturão de cisalhamento Campo do Meio na sua porção ocidental. 1993. 2 v. Tese (Doutorado) - Curso de Geologia Regional, Departamento de Petrologia e Metalogenia, Instituto de Geociências e Ciências Exatas, Universidade Estadual Paulista, Rio Claro, 1993.

MORISAWA, M; HACK, T J. ed: Tectonic geomorphology.
In: 15TH ANNUAL BINGHAMTON GEOMORPHOLOGY SYMPOSIUM, 15., 1984, Boston. Symposium. Boston: Allen And Unwin, 1985. p. 1 - 390.

PEREZ-PENA, J.V., AZANON, J.M., AZOR, A. CalHypso: an ArcGIS extension to calculate hypsometric curves and their statistical moments. Applications to drainage basin analysis in SE Spain. Computers \& Geosciences, [s.1.], v. 6, n. 6, p.12141223, 2009.

SAlAmuni, E. Tectônica da Bacia Sedimentar de Curitiba (PR). 1998. 214 f. Tese (Doutorado) - Curso de Geologia Regional, Departamento de Petrologia e Metalogenia, Universidade Estadual Paulista-Rio Claro, Rio Claro, 1998.

SCHUMM, S. A; Patton, P C. Ephemeral-stream processes: Implications for studies of Quaternary valley fills. Quaternary Research, [s.1.], v. 15, p.24-43, jan. 1981.

SCHUMM, STANLEY A. Alluvial river response to active tectonics. In: A SCHUMM, Stanley (Ed.). Active tectonics. Cambridge: Cambridge University Press, 1986. p. 80-94.

SCHUMM STANLEY A. Active Tectonics and Alluvial Rivers. In: Dumont, F J; Holbrook, J M (Ed.). Active Tectonics and Alluvial Rivers. Cambridge: Cambridge University Press, 2000. p. 276.

SCHUMM STANLEY A. Rivers and humansUnintended consequences. In: GUPTA, A. (Ed.). Large Rivers: Geomorphology and Management. Chichester: John Wiley \& Sons, 2007. p. 517-533.

SHAHZAD, FAIZAL; MAHMOOD, SYED AMER; GLOAGUEN, RICHARD. Drainage network and lineament analysis: an approach for Potwar Plateau (northern Pakistan). Journal Of Mountain Sciences, [s.1.], v. 1, n. 6, p.14-24, fev. 2009.

SHAHZAD, FAIZAL; GLOAGUEN, RICHARD. TecDEM: A MATLAB based toolbox for tectonic geomorphology, part 1: drainage network preprocessing and stream profile analysis. Comp \& Geoci, [s.1.], v. 1, n. 1, p.250-260, fev. 2011(a).

SHAHZAD, FAIZAL; GLOAGUEN, RICHARD. TecDEM: A MATLAB based toolbox for tectonic geomorphology, part 2: Surface dynamics and basin analysis. Comp \& Geoci, [s.1.], v. 1, n. 37, p.261-271, fev. 2011(b).

SIMÕES, LUÍS AMARANTE. Dobras em bainha macroscópicas relacionadas ao cisalhamento dúctil de baixo ângulo da Nappe Araxá-Canastra. Geociências, São Paulo, v. 12, n. 1, p.111-121, jan. 1993. 
SOARES, C. J. et al. Further investigation of the initial fission-track length and geometry factor in apatite fission-track thermochronology. American Mineralogist, [s.1.], v. 98, n. 8-9,p.1381-1392,1ago.a2013.aGeoScienceWorld.a

SUMMERFIELD, M. Neotectonics and landform genesis. Progress In Physical Geography, [s.1.], v. 11, n. 11, p.384-397, 1987.

SUMMERFIELD, M. Global Geomorphology: An Introduction to the Study of Landfornis. New York: John Wiley Inc, 1991. $537 \mathrm{f}$.

STEINER, SAMAR DOS SANTOS. Aquisição e processamento de dados morfométricos derivados do modelo digital de elevação SRTM. 2007. 1 v. Dissertação (Mestrado) - Curso de Geologia, Epartamento de Geologia Sedimentar e Ambiental, Universidade de São Paulo-usp, São Paulo, 2007.

STRAHLER, A N. Quantitative analysis of watershed geomorphology. Transactions Of The American Geophysical Union,Si, v. 6, n. 8, p.913-920, jan. 1957.

VALADÃO, R. C. Evolução de Longo-Termo do Relevo do Brasil Oriental (Desnudação, Superfícies de Aplainamento e Soerguimentos Crustais). 1998. 243 f. Tese (Doutorado) Curso de Geografia, Departamento de Geociências, Universidade Federal da Bahia, Salvador, 1998.

VALERIANO, C. M. et al. U-Pb geochronology of the southern Brasília belt(SE-Brazil):sedimentary provenance, Neoproterozoic orogeny and assemblyof west gondwana. Precambrian Research, [s.1.], v. 1, n. 130, p.27-55. 2004.

ZANI, H; ANDRADE FILHO, C. Hipsometria aplicada à evolução do megaleque do Taquari. In: $3^{\circ}$ SIMPÓSIO DE GEOTECNOLOGIAS NO PANTANAL, 3., 2010 , Cáceres. Anais... . Cáceres: Embrapa Informática Agropecuária/ inpe, 2010. v. 1, p. 203 - 203. 